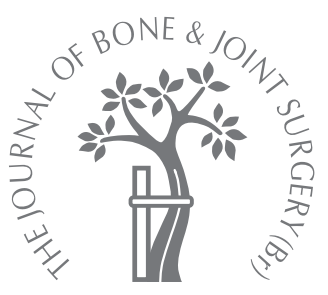

P. J. Wraighte,

P. W. Howard

From Derbyshire

Royal Infirmary,

Derby, England
P. J. Wraighte, MRCS,

Specialist Registrar in

Orthopaedics

P. W. Howard, FRCS,

Consultant Orthopaedic

Surgeon

Department of Orthopaedics Derbyshire Royal Infirmary,

London Road, Derby DE1 2OY,

UK.

Correspondence should be sent to Mr P. J. Wraighte; e-mail: pjwraighte@yahoo.co.uk

(C)2008 British Editorial Society of Bone and Joint Surgery doi:10.1302/0301-620X.90B8. $20390 \$ 2.00$

$J$ Bone Joint Surg $[\mathrm{Br}]$ 2008;90-B:1000-4. Received 25 October 2007; Accepted after revision 14 March 2008

\title{
Femoral impaction bone allografting with an Exeter cemented collarless, polished, tapered stem in revision hip replacement
}

\author{
A MEAN FOLLOW-UP OF 10.5 YEARS
}

Femoral impaction bone allografting has been developed as a means of restoring bone stock in revision total hip replacement. We report the results of 75 consecutive patients (75 hips) with a mean age of 68 years (35 to 87) who underwent impaction grafting using the Exeter collarless, polished, tapered femoral stem between 1992 and 1998.

The mean follow-up period was 10.5 years (6.3 to 14.1$)$. The median pre-operative bone defect score was 3 (interquartile range (IQR) 2 to 3 ) using the Endo-Klinik classification.

The median subsidence at one year post-operatively was $2 \mathrm{~mm}$ (IQR 1 to 3). At the final review the median Harris hip score was 80.6 (IQR 67.6 to 88.9) and the median subsidence $2 \mathrm{~mm}$ (IQR 1 to 4). Incorporation of the allograft into trabecular bone and secondary remodelling were noted radiologically at the final follow-up in $87 \%$ (393 of 452 zones) and $40 \%$ (181 of 452 zones), respectively.

Subsidence of the Exeter stem correlated with the pre-operative Endo-Klinik bone loss score $(p=0.037$ ). The degree of subsidence at one year had a strong association with longterm subsidence $(p<0.001)$. There was a significant correlation between previous revision surgery and a poor Harris Hip score $(p=0.028)$, and those who had undergone previous revision surgery for infection had a higher risk of complications ( $p=0.048$ ). Survivorship at 10.5 years with any further femoral operation as the end-point was $92 \%$ ( $95 \%$ confidence interval 82 to 97$)$.

Femoral impaction bone grafting with cement was first reported by Simon et $\mathrm{al}^{1}$ as a means of reconstituting deficient bone stock in the proximal femur in a revision total hip replacement (THR). Morcellised corticocancellous allograft was impacted within the femoral medullary canal before insertion of a collarless, polished, tapered stem with cement. Despite some satisfactory early reports, ${ }^{2}$ concerns remain over the high rate and extent of subsidence of the femoral component and the possible long-term consequences. $^{3}$

Our aim was to evaluate the long-term outcome of impaction bone grafting in revision THR using an Exeter stem (Stryker Howmedica Osteonics, Limerick, Ireland). We report our clinical and radiological results at a mean follow-up of 10.5 years $(6.3$ to 14.1$)$.

\section{Patients and Methods}

In 1992 we began using the technique of impaction bone grafting in revision THR at our institution. Between 1992 and 1998, 75 consecutive patients (40 men, 35 women; 75 hips) with a mean age of 68 years (35 to 87) underwent femoral impaction grafting during revision THR prior to cementing of an Exeter stem.

Of the 75 patients, five had at least one previous revision (Table I). A further five had been revised for an infected primary THR, as a twostage procedure.

The femoral components revised after a mean of 10.3 years from implantation $(0.5$ to 26.1) comprised of 55 Charnley (Charnley and Elite; Depuy, Leeds, United Kingdom), eight Müller (JRI, London, United Kingdom), four Exeter (Stryker Howmedica Osteonics), three Ring, two Howse, two Bateman hemiarthroplasties (3M, Loughborough, United Kingdom) and one McKee-Farrar prosthesis.

A total of 56 of the 75 operations were performed by the senior author (PWH). A posterior approach was preferred, but often the approach used for the primary procedure was utilised. After removal of the prosthesis a thorough debridement of the cement, debris and fibrous tissue was undertaken. Using a bone mill (Aesculap-Downs; Aesculap, B Braun, Sheffield, United Kingdom), cancellous chips measuring $2 \mathrm{~mm}$ to $5 \mathrm{~mm}$ in size were prepared from fresh-frozen femoral heads, previously 
Table I. Number of revisions at the initial procedure of femoral impaction allografting during revision hip replacement

\begin{tabular}{lc}
\hline Revision & Number of patients \\
\hline First & 65 \\
Second & 4 \\
Third & 1 \\
Two stage for infection & 5 \\
\hline
\end{tabular}

retrieved during primary THRs at our institution. On two occasions, irradiated and frozen femoral heads from another institution were used. A femoral restrictor was inserted $3 \mathrm{~cm}$ beyond the most distal cortical defect before impaction of the unwashed bone chips into the femoral canal using the Mark I Exeter X-change instrumentation (Stryker Howmedica Osteonics). An Exeter femoral stem was cemented into the restored intramedullary canal with either antibiotic-loaded Simplex (Stryker Howmedica Osteonics) or Palacos R (Heraeus Medical GmBH, Hanau, Germany) using the technique described by Gie et al. ${ }^{4}$

An additional calcar strut allograft was required for one proximal femur, and wire mesh for another to support the bone graft in uncontained proximal femoral defects. These were secured using cerclage wires, Dall-Miles cables (strut) and screws (mesh).

Antibiotic prophylaxis was provided by intravenous cefuroxime $(1.5 \mathrm{~g})$ at induction and two further postoperative doses of $750 \mathrm{mg}$ at intervals of eight hours. Thromboprophylaxis involved arteriovenous impulse boots and TED stockings and the administration of either adjusted low-dose warfarin or calcium heparin. Patients were mobilised as touch weight-bearing with crutches for six to eight weeks followed by progressive weight-bearing for a similar duration.

Radiological evaluation was performed on the preoperative, immediate post-operative, one-year postoperative and final follow-up visits using anteroposterior and lateral radiographs centred on the hip joint. For those patients who died during the follow-up period, the most recent radiographs before death were analysed. The radiographs were evaluated for subsidence according to the method of Fowler et al, ${ }^{5}$ radiolucencies (complete lucent lines $\geq 1 \mathrm{~mm}$ in width) according to the criteria of Gruen, McNeice and Amstutz, ${ }^{6}$ alignment of the femoral component, incorporation of bone graft including trabecular changes and healing of cortical deficiencies. All the measurements were corrected for magnification using the known dimensions of the femoral head.

The pre-operative bone loss was graded according to the Endo-Klinik classification ${ }^{7}$ (Table II). All the measurements were performed by one author (PJW) who was not the operating surgeon. The results for subsidence were calculated to provide the mean, interquartile range (IQR) and range. In order to assess reproducibility of the measure-
Table II. The Endo-Klinik grade for 75 hips at femoral impaction grafting during revision hip replacement

\begin{tabular}{lc}
\hline Endo-Klinik grade* $^{*}$ & Number of hips \\
\hline 1 & 2 \\
2 & 19 \\
3 & 50 \\
4 & 4 \\
* grade 1, radiolucent lines around upper half of \\
cement mantle; grade 2, generalised radiolucent \\
zones and endosteal erosions of the upper femur \\
leading to widening of the medullary canal; \\
grade 3, expansion of the upper femur; grade 4, \\
gross destruction of the upper end of the femur \\
with involvement of the middle third
\end{tabular}

ments, the radiographs of ten patients were re-evaluated after an interval with the investigator blinded to previous results.

The patients were reviewed annually. The Harris hip score $(\mathrm{HHS})^{8}$ was used to assess clinical outcome and those patients who lacked a recent assessment were invited to reattend. A repeat HHS was not available in 19 patients who had died and in three who had declined to attend, in two because they felt satisfied and considered it to be unnecessary and in one who was too frail. In these circumstances the most recent HHS was used.

Statistical analysis. Analysis of the data was carried out using the SPSS Version 14 software (SPSS Inc., Chicago, Illinois). Spearman's rank correlation test was used to correlate the pre-operative bone stock deficiency, subsidence of the femoral stem at one year and at the final follow-up, alignment of the femoral component, and of the HHS. Survivorship with any further reoperation as the endpoint was assessed. Subsidence over time was assessed using the Wilcoxon-signed rank test and an association with subsidence assessed using a linear regression model. The MannWhitney $U$ test was used to investigate the relationship of subsidence for the two types of cement used and the HHS. Fisher's exact test was used to test the association between complications and re-revision groups. The Shapiro-Wilk test and the Q-Q plots (SPSS Inc.) were performed to test data for a normal distribution. A p-value $\leq 0.05$ was considered to be statistically significant.

\section{Results}

The measurements were reproducible with an intra-class correlation coefficient for Endo-Klinik score of 1.00, subsidence at one year of 0.992 and subsidence at final followup of 0.990 (all measured in a subset of 10 patients). The median Endo-Klinik score for pre-operative bone loss was 3 (IQR 2 to 3) (Table II). At a mean follow-up of 10.5 years (6.3 to 14.1 ) after revision, four prostheses $(5.3 \%)$ had undergone further revision at a mean of 24 months (2 to 53 ), one for deep infection resulting in excision arthroplasty, one for a peri-prosthetic fracture managed by a nine- 


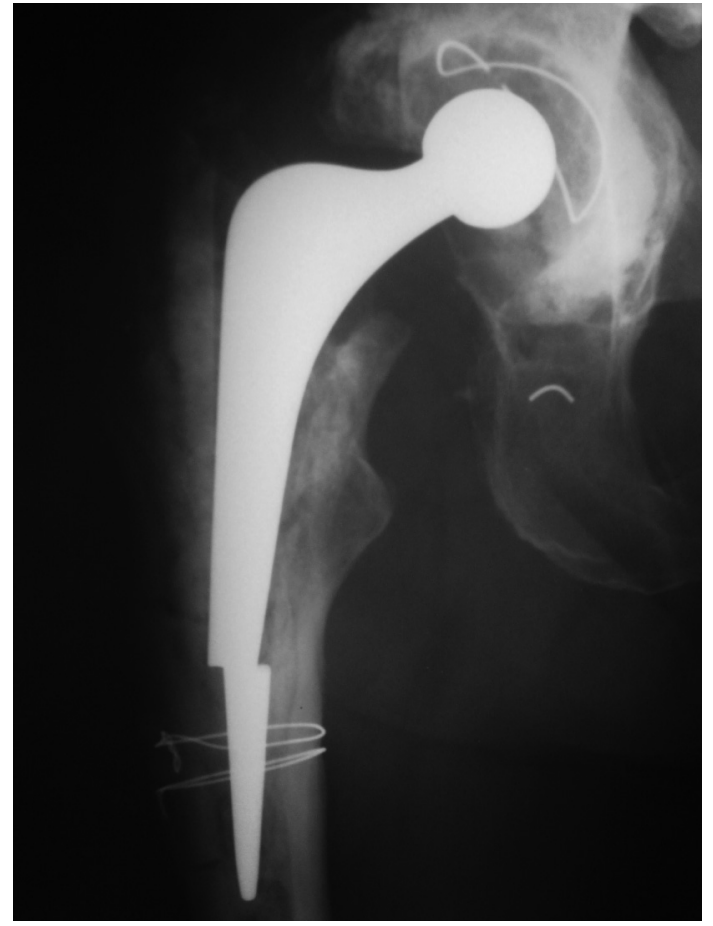

Fig. 1

Radiograph showing fracture of the femoral stem after revision hip replacement using irradiated bone graft.

hole locking plate, one for fracture of the femoral component and one for dislocation and aseptic loosening requiring a saddle prosthesis. The patient who required revision for a fracture of the femoral component (Fig. 1) had been revised for fracture of a Charnley stem at presentation. $\mathrm{He}$ was one of two patients in whom irradiated bone had been used for bone grafting and the graft had undergone resorption proximally.

In addition, another patient had a deep infection for which life-long antibiotic suppression was prescribed. The two patients complicated by infection had an HHS of 19.8 and 18.3, respectively. None of the patients previously infected who had undergone a two-stage revision were re-infected.

There were five intra-operative fractures $(6.7 \%)$, two involving the greater trochanter and three of the proximal femur all of which occurred during the removal of cement. These were all treated by cerclage wires. Two further patients developed post-operative femoral fractures, one at four weeks after inadvertently bearing weight and the other after a fall at 27 months. An intra-operative fracture was associated with greater subsidence as seen by analysis using a linear regression model $(\mathrm{p}=0.011)$.

Three patients $(4 \%)$ had post-operative dislocations, in one of whom this occurred on two occasions. One patient had a pulmonary embolus and another a deep-vein thrombosis. Two patients had Brooker Grade III heterotopic ossification. ${ }^{9}$

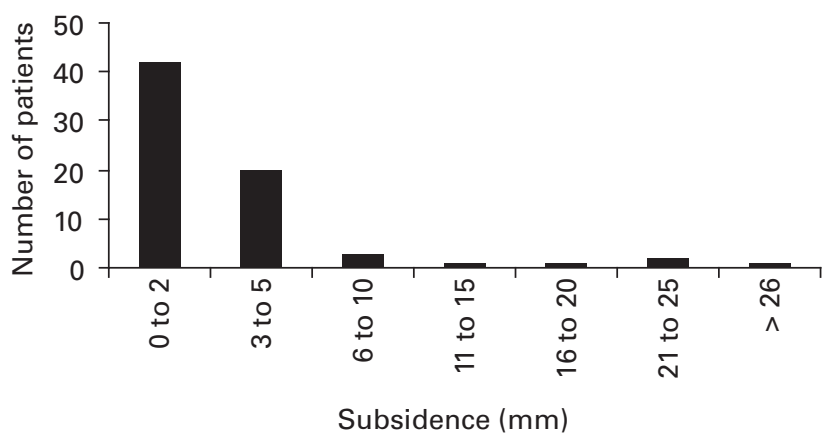

Fig. 2

Bar chart showing the incidence and extent of subsidence at the final follow-up after femoral impaction allografting during revision hip replacment.

There were no deaths in our series which were directly attributable to the revision surgery. Of the whole group, 19 patients died from unrelated causes during the follow-up period. Survivorship of the femoral component with any further femoral re-operation as the endpoint was $92 \%(95 \%$ confidence interval 82 to 97 ) at a mean of 10.5 years.

At one year the median subsidence of the femoral component was $2 \mathrm{~mm}$ (IQR 1 to 3 ) and at the final follow-up it was $2 \mathrm{~mm}$ (IQR 1 to 4 ; Fig. 2). Most of the migration occurred during the first year after revision, but there was further less marked migration during subsequent years. Five components migrated more than $10 \mathrm{~mm}$. All the femoral components subsided within the cement mantle and in one case the tip had penetrated it. The cement mantle was absent in at least one Gruen zone on the plain radiograph in two cases. There was no significant difference in the subsidence with Simplex cement compared with that of Palacos at one year after surgery (Mann-Whitney $U$ test, $p=0.295$ and 0.391 , respectively).

Initial post-operative radiographs demonstrated a mean alignment of $0.5^{\circ}$ (SD 1.1) varus. Minimal changes in alignment were observed at final follow-up, from $0^{\circ}$ to $5^{\circ}$ of valgus with only one patient moving into varus (mean change $1.4^{\circ}$ (SD 1.3) valgus). Generally, there was good evidence of reconstitution of bone stock with $87 \%$ of zones (393 of 452) demonstrating evidence of trabecular incorporation and $40 \%$ (181 of 452 ) secondary remodelling or cortical healing (Fig. 3).

Radiolucent lines were evident in 11 hips $(14.7 \%)$ at the host-graft and eight $(10.6 \%)$ of the cement-graft interface, most commonly of $1 \mathrm{~mm}$ to $2 \mathrm{~mm}$ in width and affecting Gruen zone 1. One patient had an area of cavitation extending from Gruen zones 3 to 5 .

At the most recent follow-up evaluation at a mean of 10.5 years (6.3 to 14.1) after the revision, the median HHS was 80.6 (IQR 67.6 to 88.9 ). A total of $40 \%$ of patients (25) were pain-free, $48 \%$ (29) had slight or mild pain, 7\% (4) moderate pain and 5\% (3) had severe pain and limitation. 


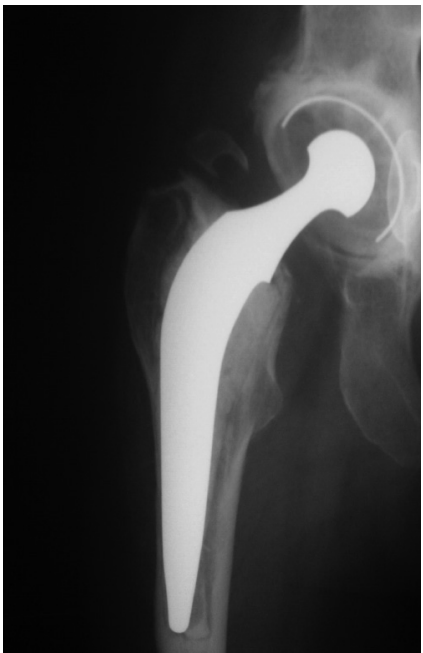

Fig. $3 a$

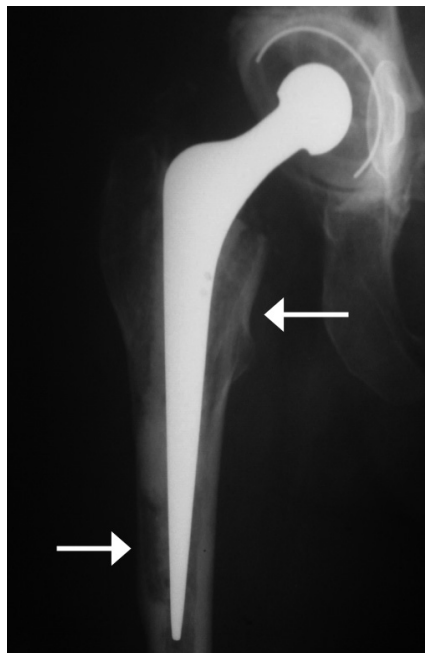

Fig. $3 b$

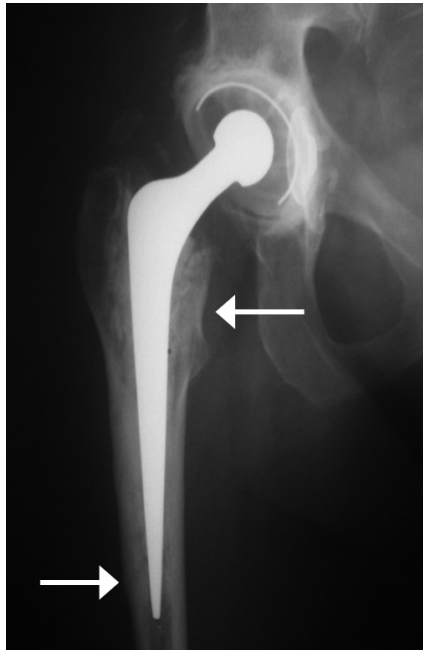

Fig. 3c

Radiographs showing femoral impaction bone allografting after revision of the femoral stem a) pre-operatively, b) post-operatively and c) at final follow-up at 11 years demonstrating trabecular incorporation and cortical healing.

The median HHS at the latest follow-up for patients who had already undergone revision before impaction allografting was 68.5 (IQR 49 to 85.2) which was significantly lower than in those undergoing revision for the first time whose median HSS was 81.8 (IQR 69.5 to 89) (MannWhitney $U$ test, $\mathrm{p}=0.028)$. Complications arose significantly more frequently for those patients who underwent re-revision for infection $(3 ; 60 \%)$ compared with those revised for aseptic loosening $(9 ; 12.9 \%)$, (Fisher's exact test, $\mathrm{p}=0.027$ ).

The pre-operative Endo-Klinik bone loss grade correlated well with the long-term subsidence of the stem ( $\mathrm{rho}=0.30$, Spearman's rank correlation, $\mathrm{p}=0.037)$. The subsidence at one year correlated highly with the long-term subsidence (rho $=0.87$, Wilcoxon signed rank, $\mathrm{p}<0.001$ ). However, the pre-operative assessment of bone stock and the extent of the subsidence were not related either to the clinical outcome as presented by the HHS or to the likelihood of complications.

\section{Discussion}

Revision THR is expensive, the functional result is not as favourable as that in primary THR, and it is associated with a higher rate of complications. ${ }^{10}$ In part this may be related to the often substantial deficiency of femoral bone stock. The technique of femoral impaction allografting with a cemented femoral stem is well documentated. ${ }^{2,11}$ Bone restoration increases revision options and may improve the mechanical stability of the implant and decrease the risk of future fractures. Although good short-term results have been reported, ${ }^{2,12}$ concern remains over the associated complication rates and the extent of subsidence of the femoral component.
Overall, the results of our series in terms of clinical outcome and survivorship of the femoral component have been good. Subsidence of the Exeter stem in femoral impaction grafting has previously raised considerable concern. Meding et $\mathrm{al}^{13}$ reported a mean subsidence of $5.3 \mathrm{~mm}$, Elting et $\mathrm{al}^{14}$ of $2.8 \mathrm{~mm}$ and Gie et $\mathrm{al}^{2}$ of $6.1 \mathrm{~mm}$ within the polymethylmethacrylate mantle using the Exeter stem. Subsidence with such prostheses is now anticipated within the first two years of primary and revision THR. ${ }^{2,5,14}$ However, Eldridge et $\mathrm{al}^{3}$ reported massive early subsidence (> $10 \mathrm{~mm}$ ) in $11 \%$ of patients using the Exeter stem. Marked subsidence has previously been thought to be associated with fracture of the cement mantle ${ }^{15}$ and to be predictive of early failure. ${ }^{16}$ The long-term consequences of such migration have previously been unknown. We report a median subsidence of $2 \mathrm{~mm}$ (IQR 1 to 3 ) at one year and of $2 \mathrm{~mm}$ (1 to 4 ) at a mean of 10.5 years. In our series five patients had subsidence in excess of $10 \mathrm{~mm}$. However, migration of the stem within the cement mantle was not found to be related to the long-term outcome or complications.

Elting et $\mathrm{al}^{14}$ reported that the subsidence ceased after two years. In our series, subsidence of the stem occurred during the first year and there was a small but statistically significant further migration over following years. The geometry of the Exeter stem allows greater migration within the cement mantle than that of other types of stem, ${ }^{17}$ but there is no associated migration between the cement and the bone in primary THR. ${ }^{18}$ At revision, cement inserted under pressure is thought to provide initial stability of the stem-cement-bone-graft construct. The collarless, polished, tapered stem allows it to subside in the cement mantle, while the cement is subjected to creep. It is believed that this allows better distribution of load, and is thought 
to provide the optimal mechanical stimulation for bone to remodel. ${ }^{4}$ The small but continued subsidence observed in our study may represent creep occurring within the cement. ${ }^{19}$ The re-orientation of the trabeculae in the grafted bone in the direction of loading and secondary trabecular remodelling observed in our study are consistent with this theory. Allograft remodelling has previously been demonstrated histologically by Ling, Timperley and $\operatorname{Linder}^{20}$ and Nelissen et al. ${ }^{21} \mathrm{~A}$ relative increase or decrease in the radiodensity of the allograft is considered to be consistent with vascular ingrowth and primary repair, ${ }^{21}$ although we acknowledge the subjective nature of this assessment and sometimes difficult interpretation of the radiographs. Of the various factors which may influence subsidence, we found that the extent of the pre-operative bone defect measured by the Endo-Klinik score had a significant correlation with long-term subsidence ( $\mathrm{p}=0.037$ ). The defects which we managed were more severe than those reported in many previous studies. ${ }^{4,13,22}$

Concern has been raised about peri-operative fractures of the femur associated with impaction grafting. ${ }^{2,14}$ In our series there were five intra-operative and two postoperative fractures. Intra-operative fractures were associated with increased subsidence of the femoral stem ( $\mathrm{p}=$ 0.011). There was no detrimental association with the longterm clinical outcome HHS or with subsequent varus/valgus malalignment.

Dislocation occurred in three patients $(4 \%)$ which was within the previously reported range of $2.3 \%$ to $11.2 \% .^{23,24}$ Deep infection occurred in two $(2.7 \%)$ of our patients following revision. This compares favourably with other studies in which the range was $2.9 \%$ to $23 \%$. $^{13,25}$

Our study showed a significant correlation between previous revisions and a poor HHS $(\mathrm{p}=0.028)$. This may relate to the extent and effect of previous surgery on the soft tissues, in particular the abductors.

We have shown good long-term clinical and radiological outcome, with a survivorship of $92 \%$ of the femoral component at a mean of 10.5 years. Subsidence continued, albeit at a lower rate, after the first year and was related to the extent of the pre-operative bone loss. Neither subsidence nor the deficiency in the bone stock was related to the long-term outcome, which was shown to correlate with a history of previous revision surgery. We recommend this technique in revision THR for patients with poor femoral bone stock.

No benefits in any form have been received or will be received from a commercial party related directly or indirectly to the subject of this article.

\section{References}

1. Simon JP, Fowler JL, Gie GA, Ling RSM, Timperley AJ. Impaction cancellous grafting of the femur in cemented total hip revision arthroplasty. J Bone Joint Surg $[B r]$ 1991;73-B(Suppl 1):73.

2. Gie GA, Linder L, Ling RSM, et al. Impacted cancellous allografts and cement for revision total hip arthroplasty. J Bone Joint Surg [Br] 1993;75-B:14-21.

3. Eldridge JD, Smith EJ, Hubble MJ, Whitehouse SL, Learmonth D. Massive early subsidence following femoral impaction grafting. J Arthroplasty 1997;12:535-40.

4. Gie GA, Linder L, Ling RS, et al. Contained morselized allograft in revision total hip arthroplasty: surgical technique. Orthop Clin North Am 1993;24:717-25.

5. Fowler JL, Gie GA, Lee AJC, Ling RSM. Experience with the Exeter Total Hip Replacement since 1970. Orthop Clin North Am 1988;19:477-89.

6. Gruen TA, McNeice GM, Amstutz HC. "Modes of failure" of cemented stem-type femoral components. Clin Orthop 1979;141:17-27.

7. Engelbrecht E, Heinnert K. Klassifikation und Behandlungsrichtlinien von Knochensubstanzverlusten bei Revisionoperationen am Hüftgelenk: mittlefristige Ergebnisse Primäré und Revisionsalloarthroplastik Hrsg. Berlin: Springer-Verlag, 1987:189-201.

8. Harris WH. Traumatic arthritis of the hip after dislocation and acetabular fractures: treatment by mold arthroplasty. J Bone Joint Surg [Am] 1969;51-A:737-55.

9. Brooker AF, Bowerman JW, Robinson RA, Riley LH. Ectopic ossification following total hip replacement: incidence and a method of classification. J Bone Joint Surg [Am] 1973;55-A:1629-32.

10. Kershaw CJ, Atkins RM, Dodd CAF, Bulstrode CJK. Revision total hip arthroplasty for aseptic failure: a review of 276 cases. J Bone Joint Surg [Br] 1991;73-B:564-8.

11. Board TN, Rooney P, Kearney JN, Kay PR. Impaction allografting in revision total hip replacement. J Bone Joint Surg [Br] 2006;88-B:852-7.

12. Pekkarinene J, Alho A, Lepisto J, et al. Impaction bone grafting in revision hip surgery: a high incidence of complications. J Bone Joint Surg [Br] 2000;82-B:103-7.

13. Meding JB, Ritter MA, Keating EM, Faris PM. Impaction bone-grafting before insertion of a femoral stem with cement in revision total hip arthroplasty. J Bone Joint Surg [Am] 1997;79-A:1834-41

14. Elting JJ, Mikhail WE, Zicat BA, et al. Preliminary report of impaction grafting for exchange femoral arthroplasty. Clin Orthop 1995;319:159-67.

15. Masterson EL, Masri BA, Duncan CP. The cement mantle in the Exeter impaction allografting technique: a cause for concern. J Arthroplasty 1997;12:759-64.

16. Walker PS, Mai SF, Cobb AG, Bentley G, Hua J. Prediction of clinical outcome of THR from migration measurements on standard radiographs: a study of cemented Charnley and Stanmore femoral stems. J Bone Joint Surg [Br] 1995;77-B:705-14.

17. Kuiper JH, Merry JC, Cheah K, et al. Early mechanical stability of impaction-grafted prostheses: effects of surgical technique, implant design and graft composition. J Bone Joint Surg [Br] 1996;78-B(Suppl II \& II):136-7.

18. Ornstein E, Frenzén $\mathbf{H}$, Johnsson $\mathbf{R}$, et al. Does the tapered shaped Exeter stem migrate at the stem-cement interface or/and at the cement-bone interface. Acta Orthop Scand 1997;68(Suppl 274):111.

19. Masterson EL, Masri BA, Duncan CP, et al. The cement mantle in femoral impaction allografting: a comparison of three systems from four centres. J Bone Joint Surg [Br] 1997;79-B:908-13.

20. Ling RSM, Timperley AJ, Linder L. Histological findings in a case of cemented femoral revision treated by endosteal impaction grafting. Acta Orthop Scand 1992;63(Suppl 248):29.

21. Nelissen RGHH, Bauer TW, Weidenhielm LR, LeGolvan DP, Mikhail PE. Revision hip arthroplasty with the use of cement and impaction grafting: histological analysis of four cases. J Bone Joint Surg [Am] 1995;77-A:412-22.

22. Halliday BR, English HW, Timperley AJ, Gie GA, Ling RS. Femoral impaction grafting with cement in revision total hip replacement: evolution of the techniques and results. J Bone Joint Surg [Br] 2003;85-B:809-17.

23. Mahoney CR, Fehringer EV, Kopjar B, Garvin KL. Femoral revision with impaction grafting and a collarless, polished, tapered stem. Clin Orthop 2005;432:181-7.

24. Engelbrecht DJ, Weber FA, Sweet MBE, Jakim I. Long term results of revision total hip arthroplasty. J Bone Joint Surg [Br] 1990;72-B:41-9.

25. Antti-Poika I, Santavirta S, Konttinen YT, Honkanen V. Outcome of the infected hip arthroplasty: a retrospective study of 36 patients. Acta Orthop Scand 1989;60:70-5. 